\title{
GENETIC ANALYSIS OF CORRELATED SEQUENTIAL CHARACTERS
}

\author{
R. L. THOMAS, J. E. GRAFIUS and S. K. HAHN* \\ Michigan State University
}

Received 1.v.70

\section{InTRODUCTION}

WHERE stresses (internal, inter-trait correlations) between sequential characters exist the more "influential" characters in the sequence may project their form of environmental and genetic control into subsequent characters through the medium of these intercorrelations. The character so affected will thus have two main determinants influencing its expression -that induced by the influence of the stresses and that operating over and above those stresses. In genetic analysis of sequential characters we generally analyse only the observed values and ignore the presence or influence of correlations. In the present paper we will subject to genetic analysis both the observed expression of a character and that part of the variation operating over and above the influence of correlations. To obtain this " remainder" variation, not ascribable to correlation, the influence of correlation as a contributory source of variance may be removed from all characters in the sequence, excluding the first, by a technique which has been previously summarised (Thomas, Grafius and Hahn, 1970a).

We have already applied the method of removing correlations to simple genotype $\times$ environment experiments (Thomas, Grafius and Hahn, 1970b). Presently we wish to extend the treatment to consideration of a more sophisticated and purely genetic analysis and will consider a situation where the environment may be presumed constant. The genetic analysis applied here - the diallel analysis of Jinks and Hayman (1953) - allows a detailed examination of the genetic system. A $4 \times 4$ diallel analysis is applied to four sequential untransformed (correlated) and transformed (uncorrelated) multiplicative characters in wheat. The four characters, which are components of yield, are in their chronological developmental sequence: heads per plant, spikelets per head, seeds per spikelet and weight/grain. Consideration is also given to progeny prediction of the complex character yield. The raw data are taken from a published source, Whitehouse, Thompson and Valle Ribeiro (1958). Both their $\mathrm{F}_{1}$ and $\mathrm{F}_{2}$ diallel sets are considered.

\section{Methods AND MATERIALS}

The experiment has been fully described by Whitehouse et al. (loc. cit.) and their material consequently needs no redescription. Transformation was applied separately to the $\mathrm{F}_{1}$ and $\mathrm{F}_{2}$ diallel sets as described by Thomas, Grafius and Hahn (1970a). The intercomponent covariances (or correlations) and variances used in the transformation were calculated from the cross mean values of the entire diallel set under consideration $\left(\mathrm{F}_{1}\right.$ or $\left.\mathrm{F}_{2}\right)$. We would have preferred utilising the correlations or covariances within

\footnotetext{
* Presently professor, Suwon University, Korea.
} 
crosses to remove the stresses within cells of the diallel table. The present procedure must therefore rest on the assumption that the inter-cell correlations are representative of the intra-cell situation and that for the latter corresponding $r$ 's are homogeneous over cells. Indirect evidence for this assumption is forthcoming from the established similarity between corresponding inter-cell $r$ 's calculated from the complete diallel and those obtained from the parental sets.

\section{TABLE 1}

Transformed and untransformed diallel cross values for the four sequential characters in wheat. Untransformed values are taken from Whitehouse et al. (1958)

\begin{tabular}{|c|c|c|c|c|c|c|c|}
\hline \multirow[b]{2}{*}{$F_{1}$ generation } & \multirow[b]{2}{*}{$\begin{array}{c}\text { Ears/Plant } \\
\text { observed }\end{array}$} & \multicolumn{2}{|c|}{ Spikelets/Ear } & \multicolumn{2}{|c|}{ Grains/Spikelet } & \multicolumn{2}{|c|}{ Weight/Grain } \\
\hline & & Observed & $\begin{array}{l}\text { Trans- } \\
\text { formed }\end{array}$ & Observed & $\begin{array}{l}\text { Trans- } \\
\text { formed }\end{array}$ & Observed & $\begin{array}{l}\text { Trans- } \\
\text { formed }\end{array}$ \\
\hline Apu; 1 & $24 \cdot 2$ & $19 \cdot 4$ & $23 \cdot 6$ & $2 \cdot 67$ & $12 \cdot 31$ & $30 \cdot 2$ & $109 \cdot 6$ \\
\hline Fram 2; 2 & $26 \cdot 5$ & $20 \cdot 2$ & $24 \cdot 8$ & $2 \cdot 13$ & $12 \cdot 26$ & $30 \cdot 6$ & $102 \cdot 3$ \\
\hline Glendowe; 3 & $19 \cdot 0$ & $21 \cdot 6$ & $24 \cdot 9$ & $2 \cdot 46$ & $12 \cdot 63$ & $35 \cdot 4$ & $105 \cdot 9$ \\
\hline Peko; 4 & $21 \cdot 7$ & $21 \cdot 6$ & $25 \cdot 4$ & $1 \cdot 69$ & $12 \cdot 05$ & $52 \cdot 4$ & $111 \cdot 0$ \\
\hline $1 \times 2$ & $27 \cdot 8$ & $20 \cdot 1$ & $24 \cdot 9$ & $2 \cdot 51$ & $12 \cdot 69$ & $32 \cdot 9$ & $113 \cdot 1$ \\
\hline $1 \times 3$ & $20 \cdot 5$ & $20 \cdot 9$ & $24 \cdot 4$ & $2 \cdot 65$ & $12 \cdot 64$ & $35 \cdot 7$ & $111 \cdot 2$ \\
\hline $2 \times 3$ & $22 \cdot 7$ & $21 \cdot 2$ & $25 \cdot 1$ & $2 \cdot 47$ & $12 \cdot 74$ & $31 \cdot 8$ & $106 \cdot 2$ \\
\hline $1 \times 4$ & $24 \cdot 4$ & $20 \cdot 8$ & $25 \cdot 0$ & $2 \cdot 11$ & $12 \cdot 33$ & $41 \cdot 4$ & $110 \cdot 7$ \\
\hline $2 \times 4$ & $24 \cdot 9$ & $21 \cdot 3$ & $25 \cdot 6$ & $2 \cdot 02$ & $12 \cdot 48$ & $41 \cdot 3$ & $109 \cdot 5$ \\
\hline $3 \times 4$ & $19 \cdot 6$ & $21 \cdot 6$ & $25 \cdot 0$ & $2 \cdot 08$ & $12 \cdot 29$ & $46 \cdot 7$ & $110 \cdot 6$ \\
\hline Average & $23 \cdot 32$ & $20 \cdot 98$ & $24 \cdot 87$ & $2 \cdot 307$ & $12 \cdot 442$ & $37 \cdot 77$ & $109 \cdot 01$ \\
\hline \multicolumn{8}{|l|}{$\mathrm{F}_{2}$ generation } \\
\hline 1 & $3 \cdot 29$ & $14 \cdot 4$ & $12 \cdot 9$ & $1 \cdot 40$ & $1 \cdot 11$ & $37 \cdot 9$ & 94.9 \\
\hline 2 & $4 \cdot 88$ & $16 \cdot 8$ & $14 \cdot 6$ & $1 \cdot 23$ & 0.99 & $35 \cdot 5$ & $89 \cdot 3$ \\
\hline 3 & $3 \cdot 24$ & $17 \cdot 7$ & $16 \cdot 2$ & $1 \cdot 46$ & $1 \cdot 02$ & $36 \cdot 6$ & $89 \cdot 5$ \\
\hline 4 & 3.76 & $16 \cdot 3$ & $14 \cdot 5$ & $1 \cdot 08$ & $0 \cdot 75$ & $58 \cdot 2$ & $99 \cdot 2$ \\
\hline $1 \times 2$ & $4 \cdot 05$ & $15 \cdot 8$ & $13 \cdot 9$ & $1 \cdot 27$ & $0 \cdot 99$ & $36 \cdot 8$ & $89 \cdot 5$ \\
\hline $1 \times 3$ & $3 \cdot 31$ & $16 \cdot 5$ & $14 \cdot 9$ & $1 \cdot 43$ & $1 \cdot 05$ & $39 \cdot 3$ & $93 \cdot 7$ \\
\hline $2 \times 3$ & $4 \cdot 30$ & $17 \cdot 4$ & $15 \cdot 4$ & 1.51 & $1 \cdot 19$ & $37 \cdot 5$ & $99 \cdot 8$ \\
\hline $1 \times 4$ & $3 \cdot 45$ & $16 \cdot 0$ & $14 \cdot 3$ & $1 \cdot 20$ & $0 \cdot 86$ & $45 \cdot 3$ & $91 \cdot 2$ \\
\hline $2 \times 4$ & $4 \cdot 18$ & $16 \cdot 4$ & 14.9 & $1 \cdot 20$ & $0 \cdot 91$ & $46 \cdot 2$ & $95 \cdot 2$ \\
\hline $3 \times 4$ & $3 \cdot 83$ & $17 \cdot 2$ & $15 \cdot 4$ & $1 \cdot 23$ & $0 \cdot 87$ & $47 \cdot 6$ & $94 \cdot 3$ \\
\hline Average & $3 \cdot 868$ & $16 \cdot 55$ & $14 \cdot 70$ & $1 \cdot 307$ & 0.974 & $42 \cdot 11$ & $93 \cdot 66$ \\
\hline
\end{tabular}

The transformed and untransformed $F_{1}$ and $F_{2}$ values are shown in table 1, and these data are subjected to the diallel graph analysis of Jinks and Hayman (1953) and the outcome shown in figs. $1 a$ to $4 b$.

For the first character in the sequence the situation is unchanged from that shown by Whitehouse et al. (loc. cit.). The remaining characters are, however, transformed and both transformed and untransformed analysis of any one character within a particular generation are included in one figure. Also included in the figures are: the limiting parabolas (dotted curve for the transformed values); the limiting points on these parabolas, indicating the point of complete additivity $\left(W r=\frac{1}{2} V p, V r=\frac{1}{4} V p\right)$ and marked by an arrow; lastly the regression line of $W r$ on $V r$, where significant, is drawn and its slope value and significance level indicated $(+=<10$ per cent.; $*<5$ per cent.; $* *<1$ per cent. and $* * *<0 \cdot 1$ per cent.) (dotted line for transformed values). 

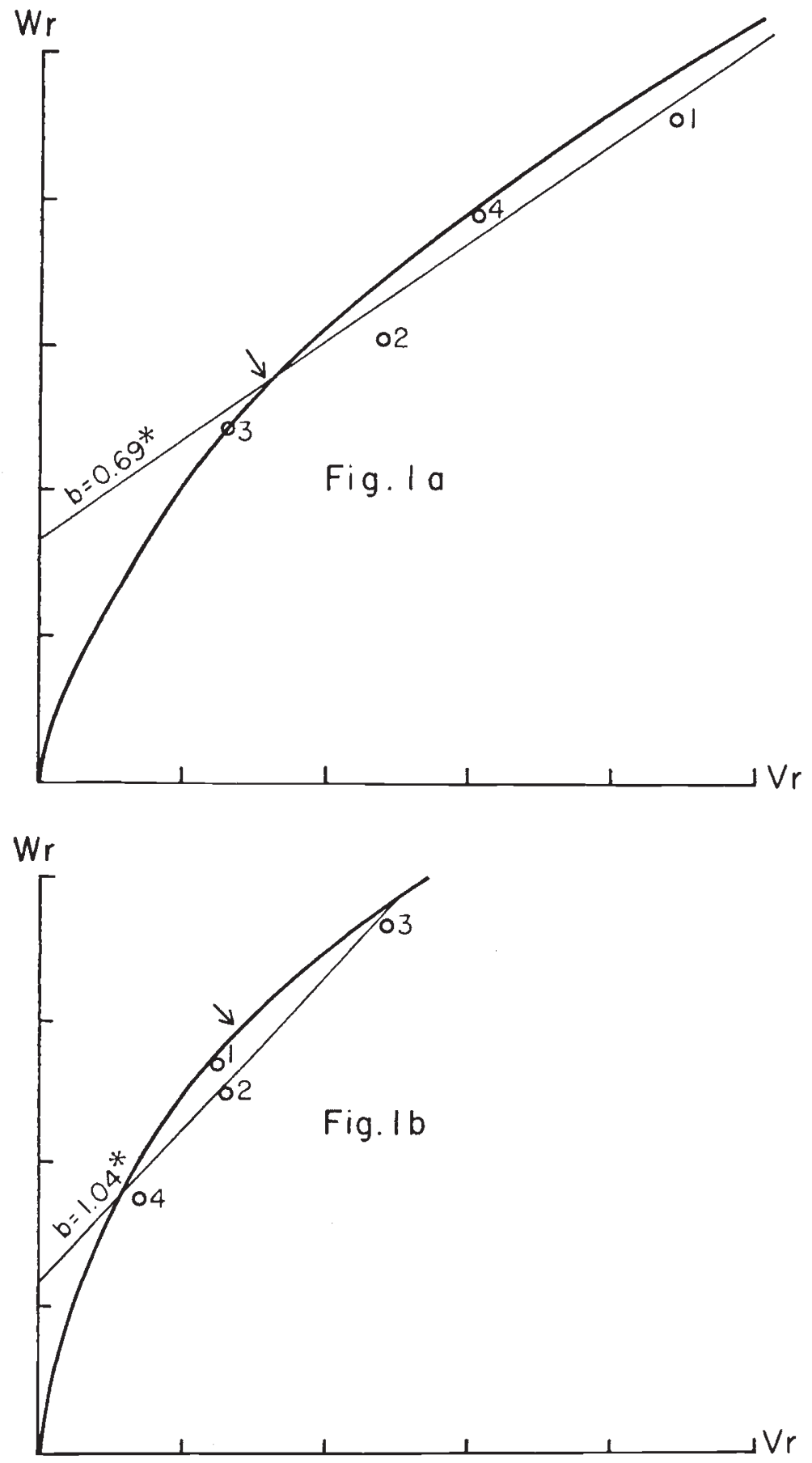

FIGs. $1 a$ and $1 b .-W r / V r$ graph analysis of heads per plant in the $\mathrm{F}_{1}(1 a)$ and $\mathrm{F}_{2}(1 b)$. 


\section{Results}

(i) Type of genetic control over the sequential characters-the $\mathrm{Wr} / \mathrm{Vr}$ graph analysis The four multiplicative characters are considered in the order of their development.

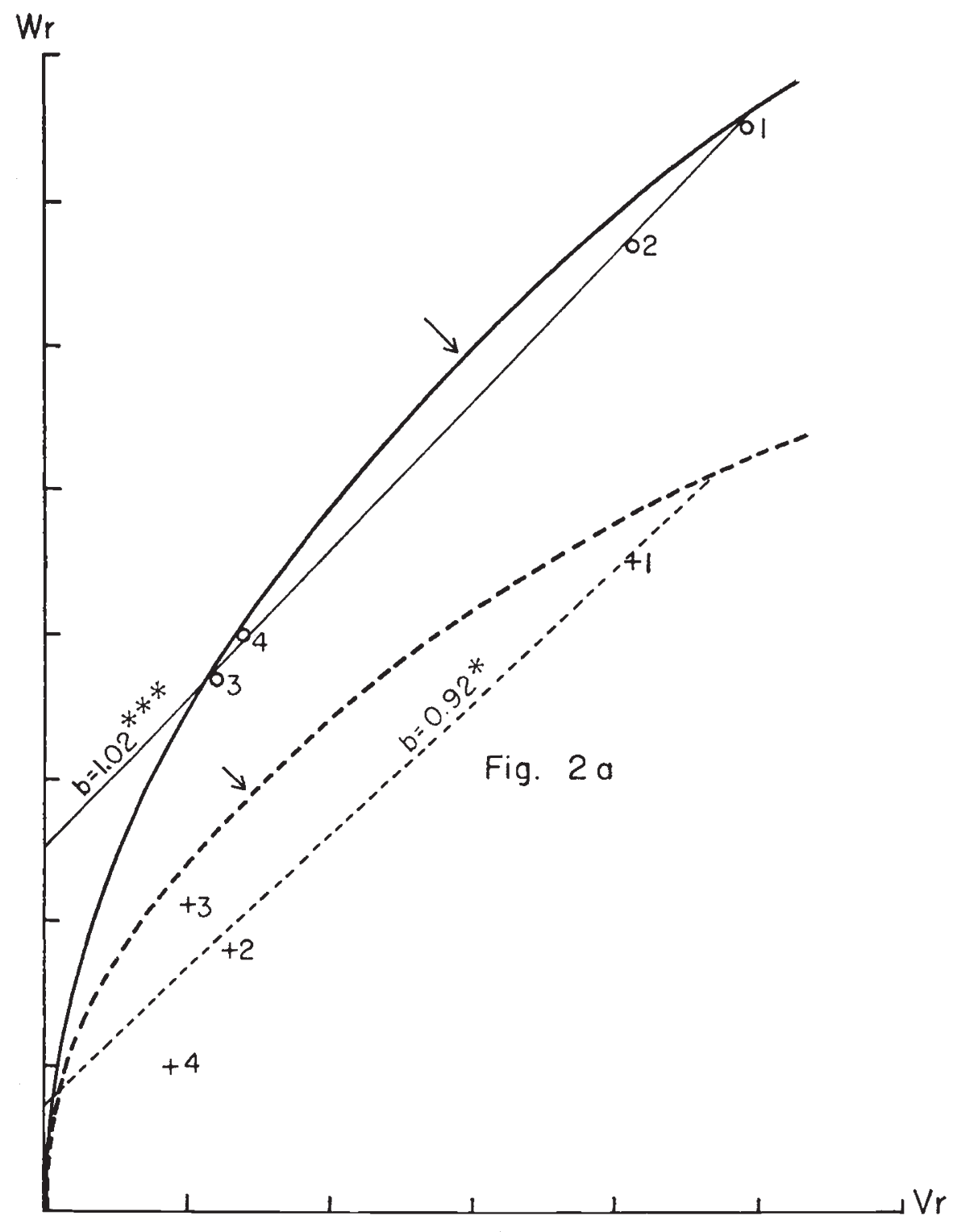

FIG. $2 a$

(a) Ears per plant. The closeness of the regression line and the four array points to the limiting parabola and limiting point in figs. $l a$ and $1 b$ indicates that little or no dominance is present for this component in either the $F_{1}$ or the $\mathrm{F}_{2}$. 
(b) Spikelets per ear. For the untransformed data in both the $\mathrm{F}_{1}$ and $\mathrm{F}_{\mathbf{2}}$ the genetic situation exhibited in figs. $2 a$ and $2 b$ again appears, from the closeness of the regression line and the array points to the parabola, to represent an additive scheme. The situation is almost unchanged from that

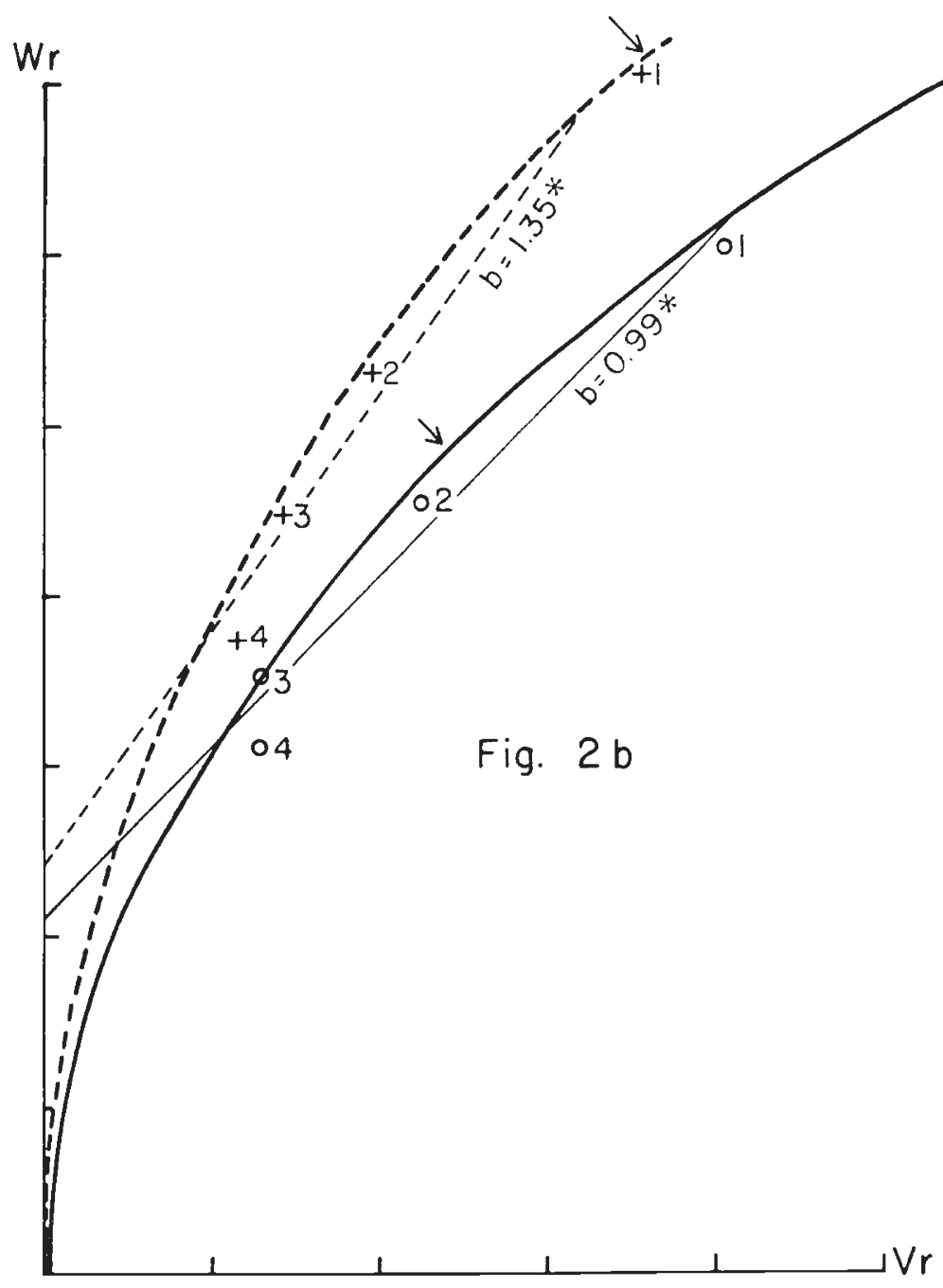

FIG. $2 b$

Figs. $2 a$ and 2b.-Wr $/ V r$ graph analysis of transformed (crosses) and untransformed (open circles) spikelets per ear in the $\mathrm{F}_{1}(2 a)$ and $\mathrm{F}_{2}(2 b)$.

of the untransformed values when the transformed $\mathrm{F}_{2}$ graph is considered. However, the graph of transformed $F_{1}$ values indicates that partial dominance is present.

(c) Grains per spikelet. The untransformed data for $\mathrm{F}_{\mathbf{1}}$ in fig. $3 a$ again indicates a highly additive genetic control. The transformed $F_{1}$ data, in the same graph, implies a high level of dominance. The situation is somewhat different in the $\mathrm{F}_{2}$, in fig. $3 b$, for both transformed and untransformed 
data. Neither regression line is significant and the pattern of distribution of the array points suggests genic interaction. This suggestion is somewhat stronger for the transformed data as may also be the mean level of dominance since the array points are generally distributed at a greater distance from the relevant limiting parabola.

(d) Weight per grain. A high level of additivity again apparently holds for untransformed data in figs. $4 a$ and $4 b$ for both generations. On the other hand, the closeness to the $V r$ axis of the array points for the transformed values in both generations could be taken as a measure of gene interaction or as an indication of a lack of genetic control over this last character in the sequence.

(e) Summary of $\mathrm{Wr} / \mathrm{Vr}$ graphs findings. The interpretation of untransformed data appears straightforward and strongly indicates that all components are under predominantly additive control. On the other hand, the transformed data appear to exhibit deviation from an additive schemetoward a higher dominance and/or genic interaction level and this tendency increases as we progress along the developmental sequence. Removing the influence of correlation appears to have revealed a somewhat different type of genetic control than that surmised from analysis of untransformed data.

\section{(ii) Degree of genetic control over the sequential characters}

There are differences in the degree as well as the differences discussed above in the type of control over these sequential characters when correlations are removed and compared with the untransformed data. This may be surmised easily from the relative positions of the limiting point in figs. $2 a$ to $4 b$. The position of this point indicates the degree of parental variance, since here $W r=\frac{1}{2} V p$ and $V r=\frac{1}{4} V p$. In turn, $V p$, the variance of parents, is a convenient measure of genetic variation (in fact, the additive variance) and therefore we must conclude that there are differences in the amount of genetic variation exhibited by a character when expressed with and without correlation. In the process of comparing the genetic variance of the transformed and untransformed values let us assume that environmental variance does not contribute, a reasonable view since it must at least be constant across transformed and untransformed values for a character and lastly assume that the narrow sense heritability of all characters, untransformed, is 100 per cent.

To compare the amount of variance present in the transformed and untransformed data we could simply express the parental variance of the former as a percentage of the latter. However, as may be seen from table 1 , the means of untransformed characters and their transformed values are different and thus the variances are not directly comparable. Therefore, in our comparison we chose to weigh each variance with its specific mean-and use this weighted variance as a measure of genetic variation. In table 2 the parental means, variances and weighted variances for transformed and untransformed characters are given. In this table the parental means and variances of the transformed data were obtained from the transformation of the parental set only and not from the complete diallel set transformation. Also, for each character a comparison of the weighted variances for transformed and untransformed data is indicated, the former being expressed as a percentage of the latter; this column is interpreted as the "amount " of genetic variance operating on the character over the apparent variance 


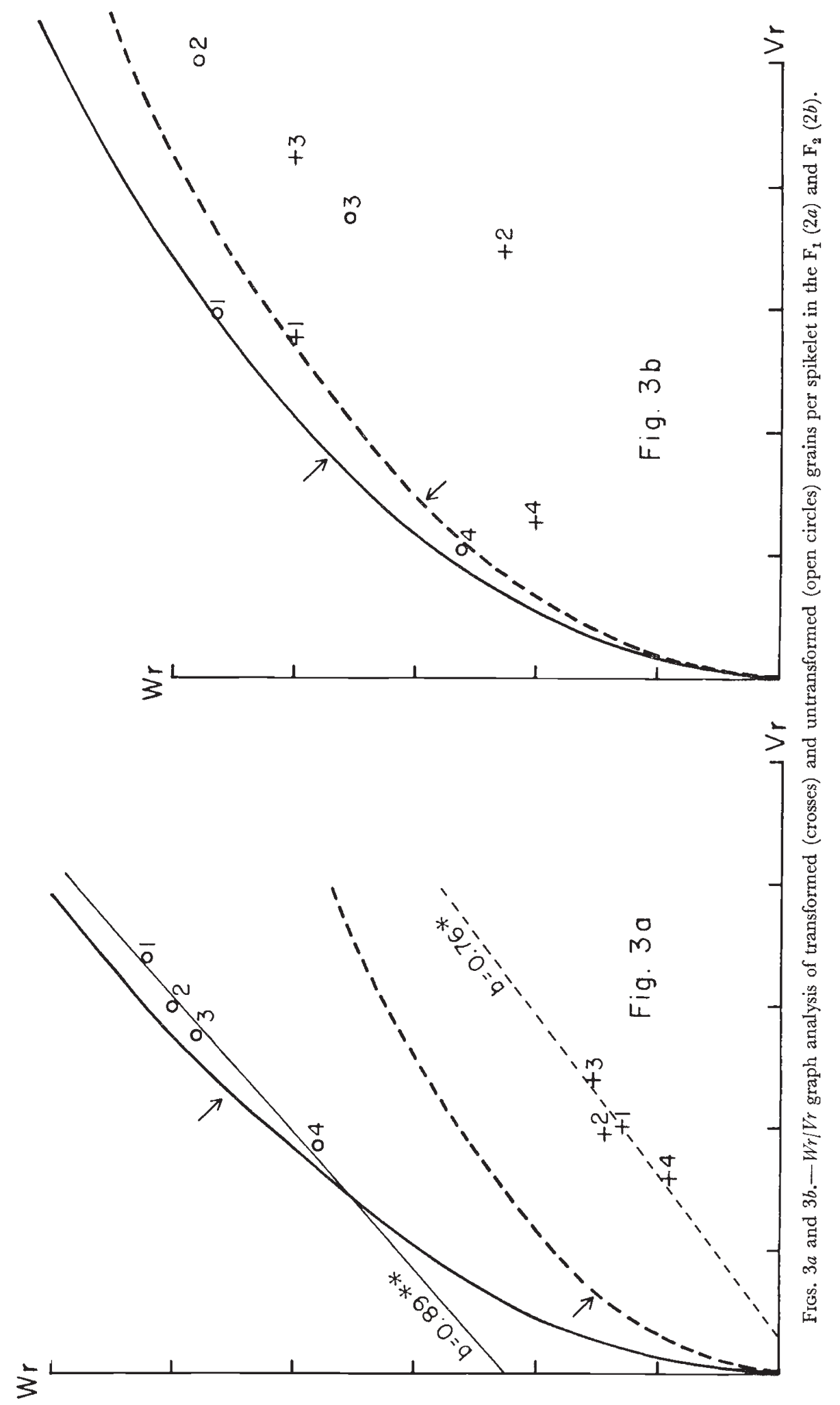


induced by the influence of correlation. Were all this genetic variance found to be additive it would also represent the "true relative" heritability of the character-but we know from the $W r / V r$ graphs that dominance is

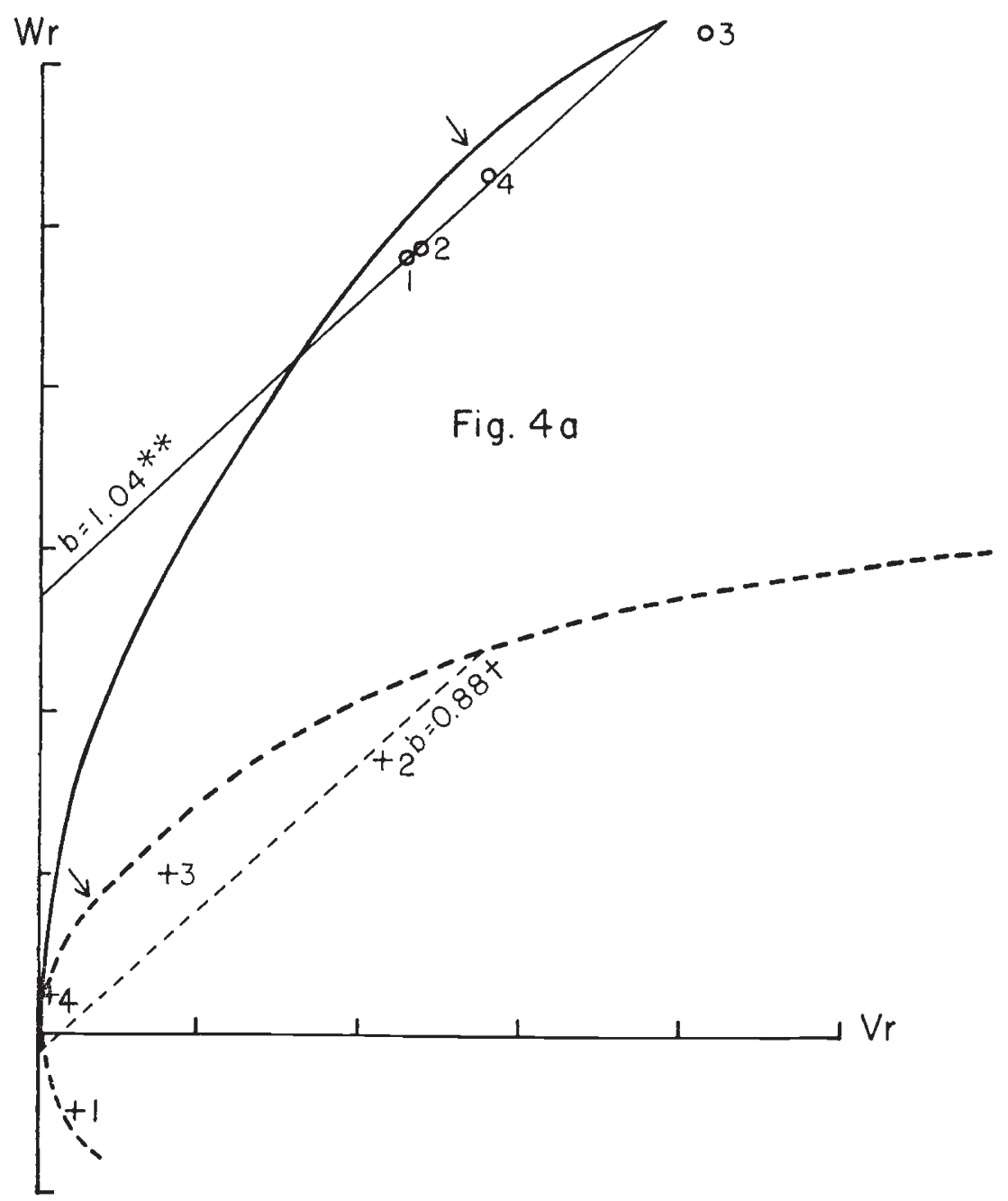

FIG. $4 a$

generally present for the transformed variable and thus the "true" heritability would generally be somewhat reduced over that indicated in the last column of table 2.

\section{(iii) The complex character, yield}

We have not subjected the complex character yield to genetic analysis since it is contended that the subject will have been covered in the analysis of the components. Whitehouse et al. (loc. cit.) did apply the $W r / V r$ graph analysis to yield and the described epistatic condition for this trait when 
compared with the additive control over its components lends some weight to Grafius (1965) position that the components may interact on a non-genic

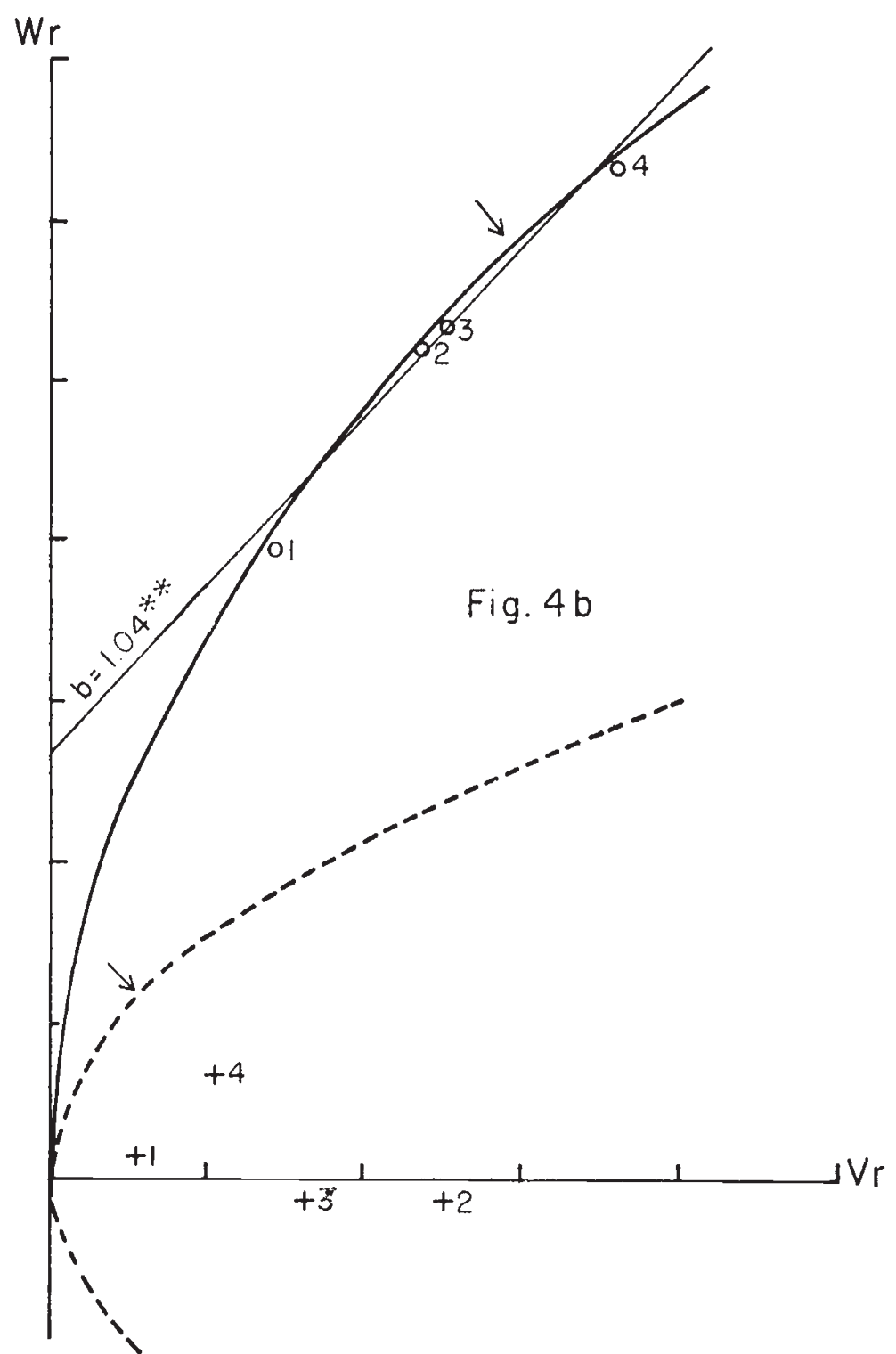

Frg. $4 b$

FIgs. $4 a$ and $4 b .-W r / V r$ graph analysis of transformed (crosses) and untransformed (open circles) weight per grain in the $F_{1}(2 a)$ and $F_{2}(2 b)$.

level. In addition, the complex inheritance pattern for yield accounts for the fairly low predictive value of midparental yield in determining progeny yield, as indicated by the low correlation values in the last column of table 3 . This is in contrast with the high predictive value of component midparents in determining their respective component expression in the progeny, which 
may be surmised from the $W r / V r$ graphs (figs. 1 to 4 ). However, naïvely resorting to the components midparents to predict progeny yield does not improve the situation. The value obtained by multiplication of component midparents only slightly improves the correlation with progeny yield in the

TABLE 2

Estimation of "true relative" genetic variance of sequential characters

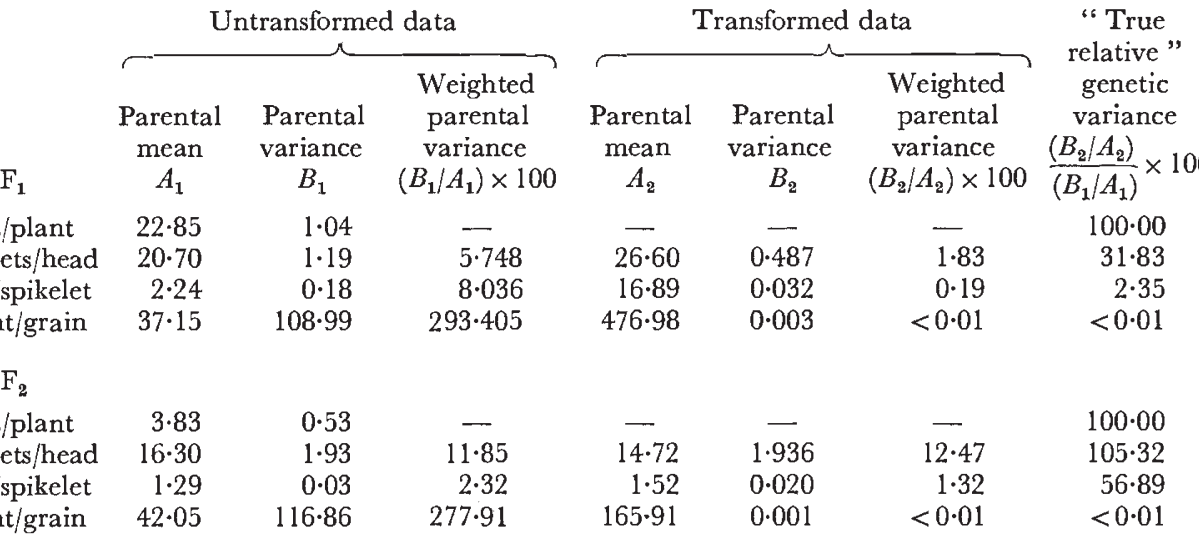

$\mathrm{F}_{2}$ but drives the correlation to virtually zero in the $\mathrm{F}_{1}$ (table 3 ). We may surmise that there is some form of intercomponent interference underlying the situation and we already have examined above the consequences of one form of such interference-the inter-trait correlations. Removal of the correlations allows the recognition of two broad categories of component:

TABLE 3

Correlation and determination coefficients of observed diallel progeny yield with calculated midparent values of individual yield components and yield in the $F_{1}$ and $F_{2}$. Yield $I$ is calculated from the midparent component values and Tield 2 directly from parental yield

\begin{tabular}{lcccccc}
\multicolumn{1}{c}{$\mathrm{F}_{1}$} & $\begin{array}{c}\text { Heads/ } \\
\text { plant }\end{array}$ & $\begin{array}{c}\text { Spikelets/ } \\
\text { ear }\end{array}$ & $\begin{array}{c}\text { Grains/ } \\
\text { spikelet }\end{array}$ & $\begin{array}{c}\text { Weight/ } \\
\text { grain }\end{array}$ & Yield 1 & Yield 2 \\
$\begin{array}{l}r(4 \text { d.f. }) \\
\text { Coefficient of } \\
\text { determination }\end{array}$ & $0.968 * *$ & -0.794 & -0.033 & -0.348 & -0.098 & 0.796 \\
$\quad \mathrm{~F}_{2}$ & $94 \%$ & $63 \%$ & $0 \%$ & $12 \%$ & $1 \%$ & $63 \%$ \\
$\begin{array}{l}r(4 \text { d.f. }) \\
\begin{array}{l}\text { Coefficient of } \\
\text { determination }\end{array}\end{array}$ & 0.449 & $0.896 *$ & -0.312 & 0.290 & 0.810 & 0.686 \\
& $20 \%$ & $80 \%$ & $10 \%$ & $8 \%$ & $66 \%$ & $47 \%$
\end{tabular}

"influencing", e.g. heads/plant in the $\mathrm{F}_{1}$ and spikelets/ear in the $\mathrm{F}_{2}$, and " influenced", e.g. the subsequent traits (see table 2). It is not too large a step to hypothesise that "influential " traits should not only strongly affect subsequent traits but contribute "disproportionately" to the variance of the complex trait. This surmise is borne out from the results given in table 3 . Here the only midparent component traits which show a significant correlation with progeny yield are, in the $\mathrm{F}_{1}$, heads/plant (94 per cent. determination) and in the $F_{2}$, spikelets/head ( 80 per cent. determination)-the influential rather than influenced characters mentioned above. 


\section{Discussion}

The "true relative" genetic variance of sequential characters when examined with the influence of previous characters in the developmental order removed differs considerably from the apparent genetic variance of the untransformed character (table 2). For the $\mathrm{F}_{1}$ diallel the actual amount of genetic variance present in the second character in the sequence, spikelets per head, is c. 30 per cent. of the apparent genetic variance and this drops off rapidly to 2 per cent. in the third character, seeds per spikelet, and to practically zero for the last, weight per grain. These values are somewhat greater than the "true" heritability estimates for these components since the analysis of the $W r / V r$ graph indicates an increase in the degree of dominance as we proceed along the sequence. Thus the true heritability differs quite considerably from the apparent (untransformed) situation. The reason for this lies in the fact that the apparent situation for spikelets/head reflects to some degree (about 70 per cent. $=100$ per cent. -30 per cent.) the observed genetic situation for heads per plant and the apparent situation for seeds/spikelet and weight/grain reflect almost completely (e.g. about 97 per cent. $=100$ per cent. -3 per cent.) the variation in the first two characters in the sequence. The mechanism of reflection is, of course, the internal correlations whose removal allows the true genetic control to be observed. In the $\mathrm{F}_{2}$ the situation is somewhat different. The last character in the sequence possesses practically no true genetic variance and the penultimate character only 55 per cent. of the untransformed variance and even here the heritability would be considerably reduced if we recall fig. $3 b$ (transformed data) where there are indications of a deviation from additivity. However, the second character in the sequence does not suffer from a reduction in "true" genetic value. In this case, however, there was little influence of heads per plant on spikelets per head though the medium of correlations, in contrast to the $\mathbf{F}_{1}$ situation. The reason for the lack of influence of heads per plant in the $\mathrm{F}_{2}$ may be surmised from table 1 , from the very low values of this character and thus its inability to produce stresssee Whitehouse et al. (loc. cit.) for an explanation of these low values in the $\mathrm{F}_{2}$. In the $\mathrm{F}_{2}$, and in the $\mathrm{F}_{2}$ only, we may conclude therefore that the true genetic situation for spikelets per head is revealed in analysis either of transformed or untransformed data and indeed the $W r / V r$ graph situation for this character does not differ greatly from transformed to untransformed data.

In summary, we may surmise that both the amount and kind of genetic variance may differ from the apparent situation when the influence of correlations are removed. The analysis of yield components illustrates the point that we might or might not be inspecting the true genetic situation when untransformed sequential characters are analysed. If the characters are strongly correlated there is good evidence that an apparent control over later characters in a sequence is merely a reflection, through stresses, of control by previous characters. It may not be a general rule that the latter characters in a sequence have little or no real direct genetic control-but this possibility will rest with analysis of other sequences. The present findings do however agree with those considered in a similar type of analysis by Thomas, Grafius and Hahn (1970b), where yield components in barley and rice were considered, in that the degree of true direct genetic control diminishes as characters further along a sequence are considered. 
The possible applications of the present findings to improving breeding techniques are rather exciting. We have already indicated in section (iii) of the Results that yield prediction is feasible in the present data by ignoring uninfluential and concentrating on one influential component. The findings may have more general application since the predictive values of midparents may be weighted with the true relative genetic variances as well as the convential heritability and multiple regression weights proposed by Grafius (loc. cit.). Such weighting applied to the present data gave a very slight improvement in determination of progeny yield over that of the best component-which is not surprising since the effectiveness of the latter is so high. What also offers some room for optimism is the finding that the type and degree of stress is under stronger environmental than genetic control (Thomas, Grafius and Hahn, 1970b), but that the expression of the primary characters in the sequence is under genetic control. Further reference to prediction will be made in a forthcoming paper (Grafius and Thomas, 1970).

\section{Summary}

1. Diallel analysis is applied to four sequential, correlated, yield component traits in wheat.

2. Analysis of the untransformed components indicates a mainly additive control of genetic variance. However, with the correlations removed deviations from additivity may be observed. These deviations increase in magnitude with progression from early to late developmental traits.

3. The parental variance of the transformed variables is generally less than the corresponding untransformed characters. This decrease becomes larger with progression along the developmental sequence away from the main influential character.

4. The degree of dropoff is more striking in the $F_{1}$ than the $F_{2}$, but in both instances the relative variance of the transformed variable compared with the untransformed variable approaches zero in the last componentseed weight.

5. A means of prediction of the complex trait, progeny yield, is suggested.

Note and Acknowledgment.-Published as article number 5002 of Michigan State University Agricultural Experiment Station. We wish to acknowledge the advice and help of Dr M. W. Adams, Dr C. M. Harrison, Dr C. Lee and Mr J. Barnard in the preparation of this manuscript.

\section{REFERENCES}

Grafius, J. E. 1965. A geometry of plant breeding. Michigan State University Res. Bull., 7. Jinks, J. L., AND hayman, B. I. 1953. The analysis of diallel crosses. Maize Genetics Coop. Newsletter, 27, 48-54.

THOMAS, R. L., GRAFIUS, J. E., AND HAHN, s. K. 1970a. Transformation of sequential quantitative characters. Heredity (in press).

THOMAs, R. L., GRAFIUS, J. E., AND HAHN, s. K. 1907b. Stress: an analysis of its source and influence. Heredity (in press).

Whitehouse, R. N. H., Thompson, J. B., AND VAlle Ribeiro, M. A. M. 1958. Studies in the breeding of self-pollinating cereals. 2. The use of diallel cross analysis in yield prediction. Euphytica, 7, 147-169. 